\title{
First report of Corynebacterium pseudotuberculosis from caseous lymphadenitis lesions in Black Alentejano pig (Sus scrofa domesticus)
}

\author{
Manuela Oliveira ${ }^{1 * \dagger}$, Cynthia Barroco ${ }^{1 \dagger}$, Carla Mottola ${ }^{1}$, Raquel Santos ${ }^{1}$, Abdelhak Lemsaddek ${ }^{2}$, Luis Tavares ${ }^{1}$
} and Teresa Semedo-Lemsaddek'

\begin{abstract}
Background: Corynebacterium pseudotuberculosis is the etiologic agent of caseous lymphadenitis, a common disease in small ruminant populations throughout the world and responsible for a significant economic impact for producers.

Case presentation: To our knowledge, this is the first characterization of C. pseudotuberculosis from caseous lymphadenitis lesions in Black Alentejano pig (Sus scrofa domesticus). In this study, phenotypic and genotypic identification methods allocated the swine isolates in C. pseudotuberculosis biovar ovis. The vast majority of the isolates were able to produce phospholipase $D$ and were susceptible to most of the antimicrobial compounds tested. Macrorestriction patterns obtained by Pulsed Field Gel Electrophoresis (PFGE) grouped the C. pseudotuberculosis in two clusters with a high similarity index, which reveals their clonal relatedness. Furthermore, swine isolates were compared with C. pseudotuberculosis from caprines and PFGE patterns also showed high similarity, suggesting the prevalence of dominant clones and a potential cross-dissemination between these two animal hosts.

Conclusions: This work represents the first report of Corynebacterium pseudotuberculosis from caseous lymphadenitis lesions in Black Alentejano pig and alerts for the importance of the establishment of suitable control and sanitary management practices to control the infection and avoid further dissemination of this important pathogen to other animal hosts.
\end{abstract}

Keywords: Antimicrobial resistance, Black Alentejano pig, Caseous lymphadenitis, Corynebacterium pseudotuberculosis, Pulsed Field Gel Electrophoresis

\section{Background}

Corynebacterium pseudotuberculosis is an important animal pathogen, being the etiological agent of caseous lymphadenitis (CLA) or cheesy gland disease in small ruminants [1]. CLA is frequently detected in major sheep and goat production areas around the world. It is characterized by abscess formation in the skin, internal and external lymph nodes and internal organs. This disease causes significant economic impact on the small ruminant industry through decreased meat yield, damaged wool and

\footnotetext{
* Correspondence: moliveira@fmv.ulisboa.pt

${ }^{\dagger}$ Equal contributors

'CIISA/Faculdade de Medicina Veterinária, Universidade de Lisboa, Avenida da Universidade Técnica, 1300-477 Lisboa, Portugal

Full list of author information is available at the end of the article
}

leather, decreased reproductive efficiency, culling of affected animals and increased morbidity and mortality rates [2]. CLA can become endemic in a herd or flock and once established it is difficult to eradicate due to its poor response to therapeutics, ability to persist in the environment and difficulties in detecting subclinical infected animals [1-3]. It is also easily spread amongst animals due to direct contact with superficial wounds or draining abscesses [3]. Nowadays, the most common treatment for CLA is abscess drainage followed by disinfection with an iodine solution and antibiotic therapy.

C. pseudotuberculosis is a relatively homogenous taxonomic group that can be distinguished from most Corynebacterium species by the production of Phospholipase 
D (PLD) and urease and the inability to ferment starch. The importance of C. pseudotuberculosis as an animal pathogen has prompted characterization studies on its toxins, particularly the haemolytic toxin PLD [4]. The differentiation between biovars is also important for infection epidemiology, as they are host specific. Evaluation of the ability to reduce nitrates allows discriminating between the negative isolates from biovar ovis, usually related with CLA in sheeps and goats, and the positive members of biovar equi, found in horses and bovines $[3,4]$.

Other species belonging to the Corynebacterium genus have already been related to CLA. Corynebacterium ulcerans was isolated from abscessed lymph nodes in wild boar (Sus scrofa) [5,6]. However, to our understanding, there is only one report available regarding C. pseudotuberculosis isolation from asymptomatic swine [7]. In this work we describe the first phenotypic and genotypic characterization of C. paratuberculosis clinical isolates from caseous lymphadenitis lesions in the Black Alentejano pig (Sus scrofa domesticus), Alentejo region, South Portugal.

\section{Case presentation}

Data presented refers to two "Alentejana" breed swine farms, A and B, located in "Odemira" district, "Alentejo" region, South Portugal, where purulent lymphadenitis cases were detected (Figure 1). In farm A and B, respectively with 700-800 and 400-500 animals, piglets are maintained in camping with the sows until weaning at 5 weeks, and afterwards are reared in extensive system, in lots with around 200 animals each. Farms only reared swine, but they were not isolated, as animals' movement could be observed. There was no commercial relationship between farms A and B. In both farms, CLA was detected in piglets from weaning until 10 month of age, in all rearing lots, affecting up to $10 \%$ of the animals. Lesions were observed mainly in the mandibular and retropharyngeal lymphnodes, but were also found in other locations. About 1\% of the animals died as a consequence of polyarthritis and/or infection dissemination to other organs. In the remaining cases, infection was controlled and was not responsible for rejections at slaughter.

Ten bacterial isolates were obtained from ten swabs collected from abscesses in ten Black Alentejano pigs (Sus scrofa domesticus). Samples were collected as part of routine diagnostics, from live animals using sterile materials and after performing the asepsis of the abscess skin, and kept refrigerated at $4^{\circ} \mathrm{C}$ until transport to the laboratory. Samples were plated on Columbia Agar (COS) (43041, bioMérieux, Basingstoke, UK) and incubated aerobically at $37^{\circ} \mathrm{C}$ for $24 \mathrm{~h}$. Isolates were initially checked for Gram staining, morphology and production of cytochrome c oxidase. From the swine samples it was possible to obtain pure cultures, formed by Gram-positive, oxidase negative, pleomorphic rods. Identification was performed using biochemical identification galleries (12136A, API Coryne, bioMérieux, Basingstoke, UK) according to the manufacturer's instructions. Subsequently, in order to distinguish between C. pseudotuberculosis biovar equi and biovar ovis, the ability to reduce nitrates was also evaluated [3]. Isolates were identified as C. pseudotuberculosis by Api Coryne ${ }^{\circ}$, all of which were nitrate reductase negative, hence identified as C. pseudotuberculosis biovar ovis. Although other microorganisms have already been related to lymphadenitis in several animals, such as Actinomyces hyovaginalis in goats, sheeps and pigs [8,9], Francisella tularensis in humans [10], Staphylococcus aureus ssp. anaerobius in lambs [11] and C. ulcerans in wild boars and roe deer [5,6], C. pseudotuberculosis is the main bacterial species responsible for CLA [1]. Results from this study confirm that this bacterial species is also the main responsible for CLA development in the Black Alentejano pig (Sus scrofa domesticus) at the sampled farms, as it was possible to isolate this agent from all collected samples, in pure cultures.

As C. pseudotuberculosis is frequently related to CLA in small ruminants but not in pigs, we included in this

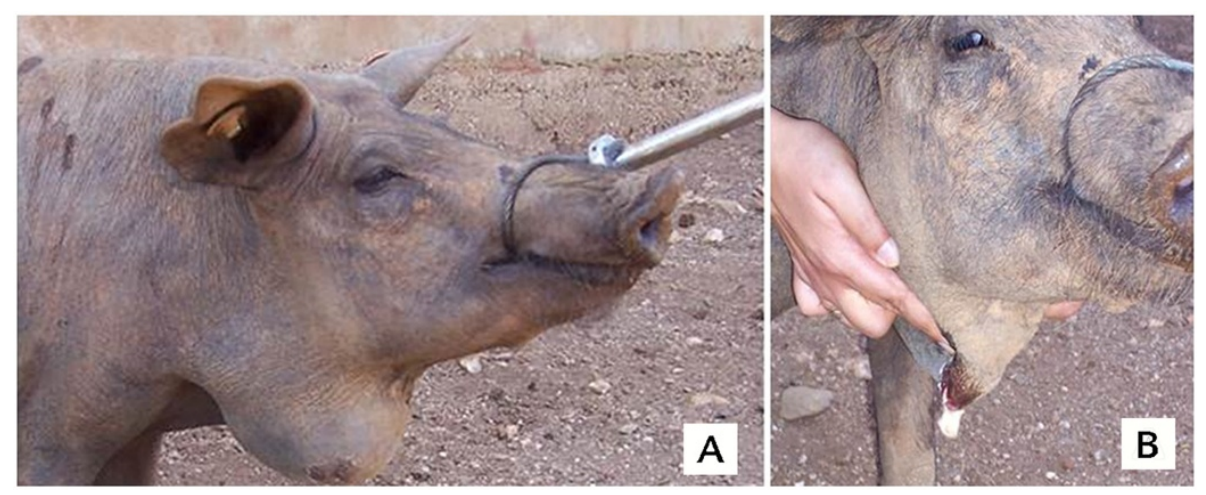

Figure 1 Caseous lymphadenitis lesions in Black Alentejano pig (Sus scrofa domesticus) from a farm located in the Odemira district, "Alentejo" region, south Portugal; A) Mandibular abscess; B) Abscess purulent content. 
study nine C. pseudotuberculosis caprine isolates belonging to a collection of clinical isolates from the Bacteriology Laboratory from the Faculty of Veterinary Medicine from the University of Lisbon, Portugal, for comparison purposes.

PLD production by the 19 isolates was determined by observation of haemolytic antagonism with $\beta$ haemolysin from Staphylococcus aureus and haemolytic synergism with Streptococcus agalactiae, according to Literák et al. [12]. cAMP phenotypic assay demonstrated that all, except one swine isolate, were PLD positive.

For assessment of the antimicrobial resistance profile, 13 antimicrobial compounds (Oxoid, Basingstoke, Hampshire, UK) commonly used in veterinary medicine were selected, as follows: Amoxycillin/Clavulanic acid (AMC, CT0223B, $30 \mu \mathrm{g}$ ), Ampicillin (AMP, CT0003B, $10 \mu \mathrm{g}$ ), Chloramphenicol (C, CT0013B, $30 \mu \mathrm{g})$, Cephalexin (CL, CT0007B, $30 \mu \mathrm{g})$, Gentamicin (CN, CT0024B, $10 \mu \mathrm{g})$, Cefotaxime (CTX, CT0166B, $30 \mu \mathrm{g}$ ), Enrofloxacin (ENR, CT0639B, $5 \mu \mathrm{g})$, Nalidixic acid (NA, CT0031B, $30 \mu \mathrm{g}$ ), Penicillin G (P, CT0043B, 10 units), Streptomycin (S, CT0047B, $10 \mu \mathrm{g})$, Sulfamethoxazole/Trimethoprim (SXT, CT0052B, $25 \mu \mathrm{g}$ ), Tetracycline (TE, CT0054B, $30 \mu \mathrm{g}$ ) and Vancomycin (VA, CT0058B, $30 \mu \mathrm{g})$. The compounds were tested by the Disk Diffusion (DD) Method, according to the Clinical and Laboratory Standards Institute [13] guidelines. Isolates were susceptible to the majority of the antimicrobial compounds tested. All C. pseudotuberculosis, except one, were resistant to nalidix acid and streptomycin. Although isolates were susceptible to most antimicrobial compounds tested, CLA infections are difficult to eradicate, as the bacterial agent remains enclosed in caseous abscesses, where the penetration of therapeutic drugs is extremely difficult.

Isolate molecular characterization was performed by Multiplex PCR and Pulsed Field Gel Electrophoresis (PFGE). For DNA isolation, 4-5 bacterial colonies of 48 hour cultures were resuspended in $100 \mu \mathrm{L}$ TE [10 mM Tris-Cl (pH 8.0), 1 mM EDTA ( $\mathrm{pH}$ 8.0)]. Afterwards, samples were incubated at $96^{\circ} \mathrm{C}$ for $5-7$ minutes and centrifuged at $15000 \mathrm{~g}$ during 5 minutes. Supernatants were stored at $-20^{\circ} \mathrm{C}$.

Primers targeting the 16S rRNA, rpoB and pld genes of C. pseudotuberculosis previously described by other authors were used [14-16]. Reference strain C. pseudotuberculosis CECT 808 was included as positive control.

Multiplex PCR was performed in a final reaction volume of $25 \mu \mathrm{L}$ containing $2.5 \mathrm{U}$ NZYTaq $2 \mathrm{x}$ Green Master Mix (NZYTech, Portugal), and $10 \mu \mathrm{M}$ of each of the primers $16 \mathrm{~S}-\mathrm{F} / 16 \mathrm{~S}-\mathrm{R}$, rpoB-F/rpoB-R and pld-F/pld-R. Reactions were carried out in a thermal cycler (MyCycler; Bio-Rad laboratories, Hemel Hempstead, UK), using the conditions described by Pacheco et al. 2007 [16].

All Corynebacterium isolates under analysis generated the amplicons of $\sim 446 \mathrm{bp}$ and $\sim 816 \mathrm{bp}$, corresponding respectively to the rpoB and $16 \mathrm{~S}$ RNA genes. Regarding the pld gene product (200 bp) it was detected in only 14 isolates. This discrepancy between PLD phenotypic and genotypic assays (18 vs. 14 positive isolates) led us to perform another PCR amplification using the primers directed to the pld gene described by Pacheco et al. but in a single reaction, i.e., $16 \mathrm{~S}-\mathrm{F} / 16 \mathrm{~S}-\mathrm{R}$, rpoB-F/rpoB-R were excluded. In this PCR amplification the 19 isolates under analysis showed a positive result. Thus, the false negative results obtained in the previous multiplex PCR were probably due to reagent consumption, which prevented pld amplification. Since the other multiplex targets were $16 \mathrm{~S}$ rRNA, rроB (two housekeeping genes present in more than one copy in the bacterial genome) this is not totally surprising. These findings highlight the need of performing both phenotypic and genotypic PLD analysis and, in case of discrepancy, converting the multiplex in a PCR directed to a single target to further confirm the negative results.

For PFGE analysis, isolates were grown in Brain-Heart Infusion broth with $0.1 \%$ Tween 20 for 48 hour at $37^{\circ} \mathrm{C}$ and DNA plugs were prepared as previously described by Connor et al. [17]. Macrorestrition was carried overnight with $20 \mathrm{U}$ of the endonuclease SfiI (Takara BIO INC, Saint-Germain-en-Laye, France) at $50^{\circ} \mathrm{C}$. PFGE was performed using a Chef DRII system (Bio-Rad laboratories, Hemel Hempstead, UK), and gels consisted of $1 \%$ agarose (Sigma-Aldrich Química, S.L) in 0.5X Tris-borateEDTA buffer (Bio-Rad laboratories, Hemel Hempstead, UK). The Lambda Ladder PFG Marker (New England Biolabs, Ipswich, USA) was used as a molecular weight marker. Electrophoresis was performed at $6 \mathrm{~V} / \mathrm{cm}$ at $14^{\circ} \mathrm{C}$ for 23 hour with an initial switch time of $5 \mathrm{~s}$ and final switch time of $20 \mathrm{~s}$. After staining with ethidium bromide, gel images were acquired with the ImageMaster (PharmaciaBiotech, GE Healthcare, UK).

BioNumerics 6.5 software (Applied Maths, Belgium) was used to register PFGE macrorestriction patterns and clustering analysis performed using the Dice similarity coefficient and the unweighted-pair group method with arithmetic mean (UPGMA).

As C. pseudotuberculosis is often related with CLA in small ruminants, goat isolates $(n=9)$ from caseous lymphadenitis lesions, belonging to a large collection of clinical isolates from the Laboratory of Microbiology and Immunology of the Faculty of Veterinary Medicine of the University of Lisbon, Portugal, were used in this study for comparison and genotypic characterization, as previously mentioned.

From the PFGE macrorestriction profiles obtained for the nineteen isolates under analysis (ten C. pseudotuberculosis from pigs and nine from goats) a similarity dendogram was built using the Bionumerics software (Figure 2). Above 55\%, isolates grouped in two clusters: cluster I, including eight swine isolates (four from each farm) and one caprine isolate; and cluster II, with eight caprine and two swine isolates (one from each farm). The higher similarity index observed 


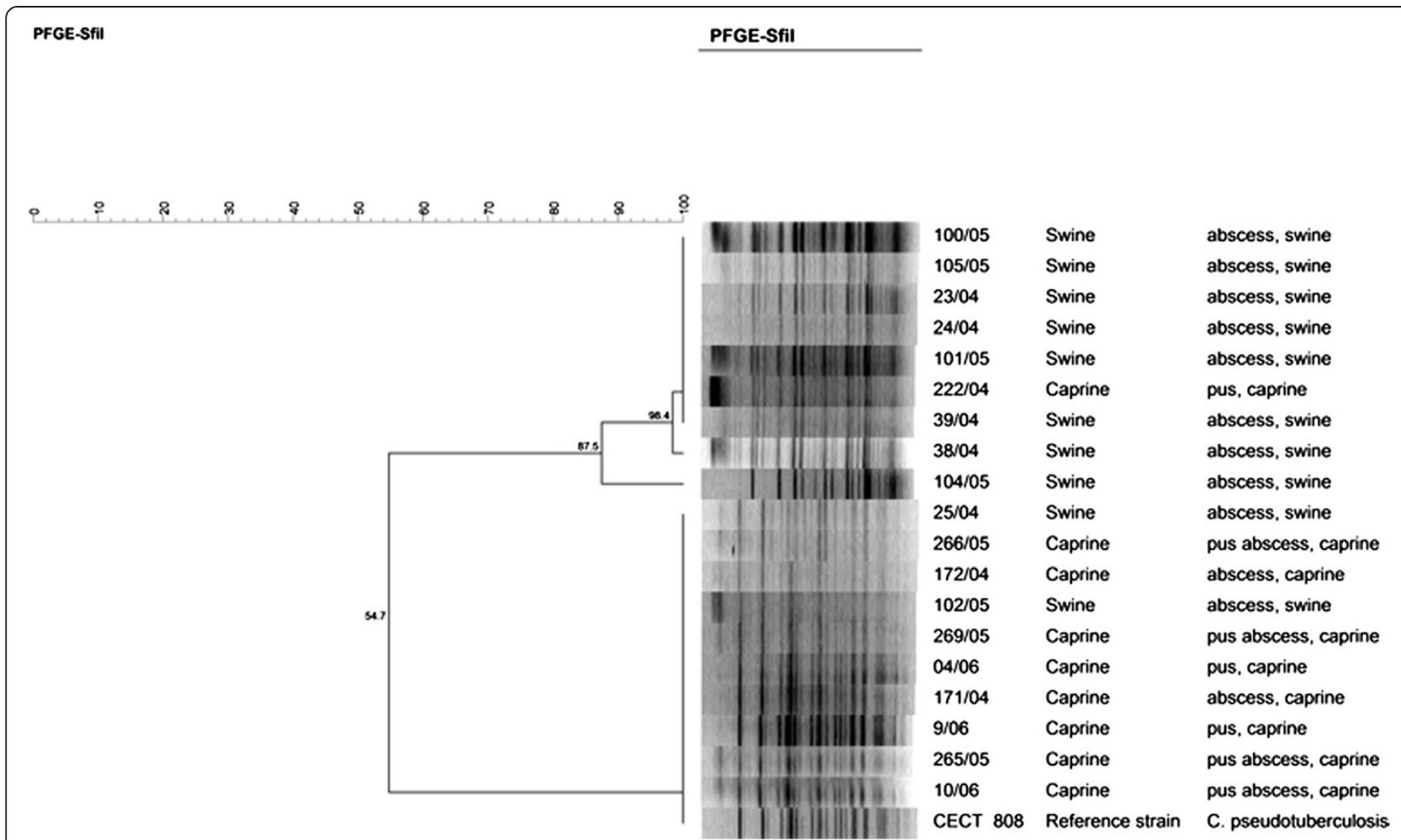

Figure 2 Dendrogram based on PFGE patterns from C. pseudotuberculosis strains analyzed using the endonuclease Sfil. Similarity was calculated with Dice correlation coefficient $-r$ - and clustering was performed with UPGMA.

within each cluster reveals the clonal relationship between the C. pseudotuberculosis under analysis, despite animal host species and farm of origin. Similar results were reported by other authors who also found high genomic similarities between C. pseudotuberculosis isolated from distinct animal species $[3,17,18]$.

Overall, our study suggests the prevalence of dominant clones and a putative cross-dissemination between swine and goats. As the farms under study only rear this swine breed, animals could have been infected by pigs coming from other farms that also have sheep and goats, as the production system was not closed.

\section{Conclusions}

This work represents the first report of Corynebacterium pseudotuberculosis from caseous lymphadenitis lesions in Black Alentejano pig (Sus scrofa domesticus). It also alerts for the importance of the establishment of suitable control and sanitary management practices to control the infection and avoid further dissemination of this important pathogen to other animal hosts. The presence of this microorganism in the two farms studied confirms its spreading ability, as well as their clonal relationships established by PFGE analysis. Further studies including a larger number of isolates should be performed in order to fully characterize these agents and identify possible routes of transmission.

\section{Competing interests}

Authors have no financial or non-financial competing interests to declare.

\section{Authors' contributions}

$\mathrm{MO}$ conceived the study and participated in its coordination, helped to draft the manuscript, having also participated in bacterial isolation and in the phenotypic assays; CB carried out the phenotypic assays and the molecular genetic studies; CM participated in the molecular genetic studies; RS participated in results analysis and helped to draft the manuscript; $\mathrm{AL}$ participated in the sequence alignment; LT participated in the study design and coordination; TSL participated in the design, coordination and execution of the molecular genetic studies and helped to draft the manuscript. All authors read and approved the final manuscript.

\section{Acknowledgements}

Authors would like to acknowledge Lina Cavaco, DVM, PhD and Filipe Nunes, DVM, PhD, for all the help in isolates collection and identification and Diogo Costa, DVM, for allowing access to the farms. This work was supported by CIISA ("Centro de Investigação Interdisciplinar em Sanidade Animal") from the Faculty of Veterinary Medicine, University of Lisbon, Project PEst-OE/AGR/U10276/2014, funded by "Fundação para a Ciência e Tecnologia" (FCT), Lisbon, Portugal.

\section{Author details}

${ }^{1}$ CIISA/Faculdade de Medicina Veterinária, Universidade de Lisboa, Avenida da Universidade Técnica, 1300-477 Lisboa, Portugal. ${ }^{2}$ BioFIG, Centro para a Biodiversidade, Genómica Integrativa e Funcional, Faculdade de Ciências, Universidade de Lisboa, 1749-016 Lisboa, Portugal. 
Received: 8 May 2014 Accepted: 16 September 2014

Published online: 21 September 2014

\section{References}

1. Dorella FA, Pacheco LC, Oliveira SC, Miyoshi A, Azevedo V: Corynebacterium pseudotuberculosis: microbiology, biochemical properties, pathogenesis and molecular studies of virulence. Vet Res 2006, 37:1-18.

2. Williamson LH: Caseous lymphadenitis in small ruminants. Vet Clin North Am Food Anim Pract 2001, 17:359-371.

3. Soares SC, Silva A, Trost E, Blom J, Ramos R, Carneiro A, Ali A, Santos AR, Pinto AC, Diniz C, Barbosa EG, Dorella FA, Aburjaile F, Rocha FS, Nascimento KK, Guimarães LC, Almeida S, Hassan SS, Bakhtiar SM, Pereira UP, Abreu VA, Schneider MP, Miyoshi A, Tauch A, Azevedo V: The pan-genome of the animal pathogen Corynebacterium pseudotuberculosis reveals differences in genome plasticity between the biovar ovis and equi strains. PLOS ONE 2013, 8:e53818.

4. Coyle MB, Lipsky BA: Coryneform bacteria in infectious diseases: Clinical and laboratory aspects. Clin Microbiol Rev 1990, 3:227-246.

5. Contzen M, Sting R, Blazey B, Rau J: Corynebacterium ulcerans from Diseased Wild Boars. Zoonoses Public Health 2011, 58:479-488.

6. Eisenberg T, Kutzer P, Peters M, Sing A, Contzen M, Rau J: Nontoxigenic tox-bearing Corynebacterium ulcerans infection among game animals, Germany. Emerg Infect Dis 2014, 20:448-452.

7. Zhao HK, Yonekawa K, Takahashi T, Kikuchi N, Hiramune T, Yanagawa R: Isolation of Corynebacterium pseudotuberculosis from the cervical canal of clinically normal sows. Res Vet Sci 1993, 55:356-359.

8. Schumacher VL, Hinckley L, Gilbert K, Risatti GR, Londoño AS, Smyth JA: Actinomyces hyovaginalis-associated lymphadenitis in a Nubian goat. J Vet Diagn Invest 2009, 21:380-384.

9. Foster G, Wragg P, Koylass MS, Whatmore AM, Hoyles L: Isolation of Actinomyces hyovaginalis from sheep and comparison with isolates obtained from pigs. Vet Microbiol 2012, 157:471-475

10. Markoc F, Koseoglu RD, Koc S, Gurbuzler L: Tularemia in differential diagnosis of cervical lymphadenopathy: cytologic features of tularemia lymphadenitis. Acta Cytol 2014, 58:23-28.

11. Møller K, Agerholm JS, Ahrens P, Jensen NE, Nielsen TK: Abscess disease, caseous lymphadenitis, and pulmonary adenomatosis in imported sheep. J Vet Med B Infect Dis Vet Public Health 2000, 47:55-62.

12. Literák I, Horváthová A, Jahnová M, Rychlík I, Skalka B: Phenotype and genotype characteristics of the Slovak and Czech Corynebacterium pseudotuberculosis strains isolated from sheep and goats. Small Rumin Res 1999, 32:107-111.

13. Clinical and Laboratory Standards Institute: Performance Standards for antimicrobial Susceptibility Testing; Seventeenth Informational Supplement. In CLSI document M100-S17; 2007.

14. Çetinkaya B, Karahana M, Atila E, Kalina R, De Baereb T, Vaneechoutteb M: Identification of Corynebacterium pseudotuberculosis isolates from sheep and goats by PCR. Vet Microbiol 2002, 88:75-83.

15. Khamis A, Raoult D, La Scola B: rpoB gene sequencing for identification of Corynebacterium species. J Clin Microbiol 2004, 42:3925-3931.

16. Pacheco LG, Pena RR, Castro TL, Dorella FA, Bahia RC, Carminati R, Frota MN, Oliveira SC, Meyer R, Alves FS, Miyoshi A, Azevedo V: Multiplex PCR assay for identification of Corynebacterium pseudotuberculosis from pure cultures and for rapid detection of this pathogen in clinical samples. J Med Microbiol 2007, 56:480-486.

17. Connor KM, Fontaine MC, Rudge K, Baird GJ, Donachie W: Molecular genotyping of multinational ovine and caprine Corynebacterium pseudotuberculosis isolates using pulsed-field gel electrophoresis. Vet Res 2007, 38:613-623.

18. Dorneles EM, Santana JA, Ribeiro D, Dorella FA, Guimarães AS, Moawad MS, Selim SA, Garaldi AL, Miyoshi A, Ribeiro MG, Gouveia AM, Azevedo V, Heinemann MB, Lage AP: Evaluation of ERIC-PCR as genotyping method for Corynebacterium pseudotuberculosis isolates. PLOS ONE 2014, 9:e98758.

doi:10.1186/s12917-014-0218-3

Cite this article as: Oliveira et al:: First report of Corynebacterium pseudotuberculosis from caseous lymphadenitis lesions in Black Alentejano pig (Sus scrofa domesticus). BMC Veterinary Research 2014 10:218.

\section{Submit your next manuscript to BioMed Central and take full advantage of:}

- Convenient online submission

- Thorough peer review

- No space constraints or color figure charges

- Immediate publication on acceptance

- Inclusion in PubMed, CAS, Scopus and Google Scholar

- Research which is freely available for redistribution

Submit your manuscript at www.biomedcentral.com/submit
C BioMed Central 